\title{
A Microscopic Account of Black Hole Entropy
}

\section{String theory provides a microscopic description of the entropy of certain theoretical black holes-an important step toward understanding black hole thermodynamics.}

by Leopoldo A. Pando Zayas ${ }^{1,2}$

$\mathrm{I}$ $\mathrm{n}$ the 1970's, theorists determined that black holes have entropy [1], a remarkable finding that points at analogies between these spacetime singularities and systems 1 of particles, such as classical gases. The crucial proof was provided by Stephen Hawking, who demonstrated, using a quantum-mechanical framework, that black holes radiate as if they were black bodies with a specific temperature [2]. The analogy was completed by extending all four laws of thermodynamics to black holes [3]. In thermodynamics, entropy is an important bridge between the macroscopic and the microscopic world: In a gas, for instance, entropy relates macroscopic heat transfer to the number of available

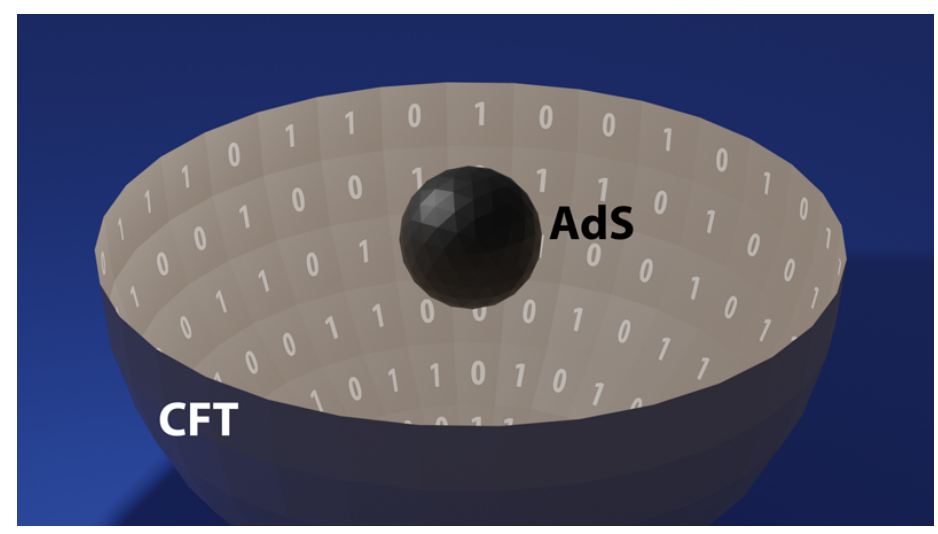

Figure 1: Through the AdS/CFT correspondence, a black hole in a universe with gravity (AdS) can be represented in terms of a dual system described by a quantum field (CFT). Exploiting this correspondence, Benini and Milan have been able to compute a black hole's entropy starting from microscopic quantum degrees of freedom [4]. (APS/Alan Stonebraker)

\footnotetext{
${ }^{1}$ Leinweber Center for Theoretical Physics, University of Michigan, Ann Arbor, MI, USA

${ }^{2}$ The Abdus Salam International Centre for Theoretical Physics, Trieste, Italy
}

microscopic states of the gas molecules. Providing a similar microscopic explanation of black hole entropy is an important test for theories that aim to unify gravity and quantum mechanics. Now, three independent studies have shown that string theory can offer a microscopic accounting of entropy for certain theoretical black holes [4-6]. The result sets up these black holes as ideal toy models for running thought experiments that tackle subtle questions of quantum gravity.

Providing a statistical mechanics interpretation of black hole entropy has been a long-standing goal for candidate quantum-gravity theories. To make calculations tractable, researchers examine theoretical black holes that provide an idealized description of real astrophysical ones. In 1996, physicists Andrew Strominger and Cumrun Vafa showed that string theory can provide such an interpretation [7]. Through spectacularly sophisticated calculations, the duo showed that the entropy of certain supersymmetric black holes contemplated in string theory could be computed by counting the states of stringy degrees of freedom known as D-branes-multidimensional objects that, in string theory, replace point particles. Despite providing an important consistency check for string theory, this result hasn't led to the resolution of important theoretical puzzles related to black hole physics.

Around the same time, physicist Juan Maldacena offered a new approach for tackling quantum gravity problems through the so-called anti-de Sitter/conformal field theory (AdS/CFT) correspondence [8]. Maldacena's conjecture states that for a certain quantum system with gravity there is an equivalent system without gravity. This equivalence allows a universe with gravity to be studied by modeling it with a simpler, gravity-free universe described by a quantum field theory.

The correspondence implicitly contains the resolutions of many gravitational puzzles, including what is known as the black hole information paradox. The idea originated with Hawking, whose calculations suggested that evaporating black holes can destroy information about the quantum states of particles that fell into them. This conclusion, however, is incompatible with the laws of quantum mechanics, which says that a particle's wave function at one time determines its value at any future time. However, 
a workaround is provided by the so-called AdS/CFT correspondence, which states there can be a dual quantum system equivalent to a system with gravity. In this dual system, the formation and evaporation of black holes can be described by quantum field equations that don't allow information loss. Largely based on this argument, Hawking admitted that black hole evaporation does in fact preserve information, conceding a bet he made 30 years earlier. While AdS/CFT implicitly solves the paradox, it hasn't yet produced a full, explicit explanation of the thermodynamic properties of black holes.

The three new studies-by Francesco Benini and Paolo Milan of the International School for Advanced Studies (SISSA) and Abdus Salam International Centre for Theoretical Physics (ICTP), both in Italy [4], by Alejandro Cabo-Bizet of King's College London and colleagues [5], and by Sunjin Choi of Seoul National University, South Korea, and colleagues [6]-make important contributions to filling this gap. Exploiting the AdS/CFT correspondence, the three teams successfully perform a microstate counting for certain theoretical black holes in the dual AdS space [4-6]. In this space, rotating, electrically charged, and five-dimensional black holes are described by a quantum field theory similar to quantum chromodynamics-the theory that describes the interaction of quarks and gluons. From the microscopic degrees of freedom identified by this quantum field theory, the researchers compute the partition function that describes the statistical properties of the microstates and is related to thermodynamic variables, including entropy.

The derivations of the three groups were enabled by the same mathematical trick. Namely, the researchers allow chemical potentials of the particles in the field theory to take on complex values, rather than only real ones. (The chemical potential of a system of particles is defined as the freeenergy change resulting from the addition or subtraction of a particle.) The entropy calculated from this microscopic accounting was found to exactly match the well-known formula for the macroscopic entropy of the dual black hole. Although the groups took similar approaches, Cabo-Bizet et al. and Choi et al. restricted their calculations to black holes that spin infinitely fast, while Benini and Milan relaxed this assumption to include all spin rates, which makes their results more general and more closely related to astrophysical situations.

Since the three studies were first posted on the arXiv in 2018, they have triggered research efforts that have confirmed the robustness of the new approach. Similar compu- tations have successfully provided a microscopic accounting of the entropy for AdS black holes in 4 to 7 dimensions and for slightly nonsupersymmetric black holes. Other work has shown that this microscopic accounting can exactly predict certain quantum corrections to entropy for systems than can be solved in both the AdS system and its CFT dual system [9].

The three teams have completed the important step of identifying the microscopic degrees of freedom responsible for these black holes' entropy. Since the approach is based on degrees of freedom that are familiar to other fields of physics, it will invite fresh contributions from many researchers, such as those working on lattice field theory and condensed-matter theory. A natural continuation of the work would be to move from mathematical aspects of state counting to deeper questions of black hole dynamics. If the new microscopic approach can provide an explicit, nutsand-bolts derivation of the rate of Hawking radiation, it will have answered one of the key questions of black hole dynamics and possibly provided the ultimate resolution of the information paradox.

This research is published in Physical Review X, in the Journal of High Energy Physics, and posted on the arXiv.

\section{REFERENCES}

[1] J. D. Bekenstein, "Black holes and entropy," Phys. Rev. D 7, 2333 (1973).

[2] S. W. Hawking, "Particle creation by black holes," Commun. Math. Phys. 43, 199 (1975).

[3] J. M. Bardeen et al., "The four laws of black hole mechanics," Commun. Math. Phys. 31, 161 (1973).

[4] F. Benini and P. Milan, "Black holes in 4D $\mathcal{N}=4$ Super-YangMills field theory," Phys. Rev. X 10, 021037 (2020).

[5] S. Choi et al., "Large AdS black holes from QFT," arXiv:1810.12067.

[6] A. Cabo-Bizet et al., "Microscopic origin of the BekensteinHawking entropy of supersymmetric AdS5 black holes," J. High Energy Phys. 2019, 62 (2019).

[7] A. Strominger and C. Vafa, "Microscopic origin of the Bekenstein-Hawking entropy," Phys. Lett. B 379, 99 (1996).

[8] J. Maldacena, "The large $\mathrm{N}$ limit of superconformal field theories and supergravity," Int. J. Theor. Phys. 38, 1113 (1999).

[9] F. Benini et al., "Rotating black hole entropy from M5-branes," J. High Energy Phys. 2020, 57 (2020).

10.1103/Physics. 13.80 